\title{
Note on Editing the Texts
}

Laycock's 'Account' is a complex manuscript. It is an autobiographical narrative of his election to the professorship of the Practice of Physic at Edinburgh University in 1855. It also contains Laycock's reflections on a subsequent dispute about the teaching of clinical medicine at the Royal Infirmary of Edinburgh (1857), as well as a discussion of the controversy surrounding the election of a new University Principal in 1868. Laycock's narrative is reproduced in its entirety here and is referred to throughout as the Main Text.

The manuscript also contains a variety of original sources bearing upon these three episodes which Laycock collected, inserted and made reference to throughout the Main Text. These are located and described using footnotes. A $\{t\}$ and/or a $\{p\}$ in a footnote indicates a source is transcribed and/or reproduced photographically in one of three supplementary texts. Supplementary Text 1 consists of correspondence, Supplementary Text 2, miscellaneous documents, and press cuttings comprise Supplementary Text 3. As documents in the supplementary text are organised chronologically, each transcription is preceded by a cross-reference to its original location in the Main Text.

With the exception of two letters and one telegram which Laycock copied directly into his narrative, all the holograph and other letters are transcribed in Supplementary Text 1. A selection of printed addresses, voting lists and various notes make up Supplementary Text 2. Some which did not lend themselves easily to exact transcription are reproduced photographically. Approximately half of the original press cuttings are transcribed in Supplementary Text 3 . In the few cases where Laycock did not date a press cutting, or he was unsure which newspaper it came from, it is not always possible to give precise references for these sources.

Two minor documents affixed to blank pages before the Main Text commences relate to Laycock's student days at University College London and have been omitted. Some illustrations, and those loose cuttings and articles found with the volume which date after Laycock's death were also excluded. It should also be noted that Laycock occasionally referred to an additional "letter folio", and extracts from Edinburgh University Faculty of Medicine Minutes, neither of which has been found. As well as the original sources, a variety of additional illustrations have been included, such as photographs of Laycock and some of the Edinburgh medical men who figure prominently in the 'Account', sample pages of the original manuscript and title pages of some of his other writings referred to in the Main Text.

Throughout all texts a [?] indicates the transcription of words or phrases is uncertain; a [...] that they are illegible. In addition a $\{b\}$ is used to indicate that further information about an individual mentioned by Laycock is available in the Biographical List. In the Main Text abbreviations are expanded, surnames standardised, punctuation and capitalization modernised, notes and marginalia integrated into the Main Text. In the supplementary texts, however, original sources are always transcribed as exactly as possible. Any annotations Laycock made to them are indicated by \{Annotation:\} with the relevant addition in inverted commas inside this form of brackets. All references to the 
'Account' indicated by the abbreviation (A) refer to page numbers in this edition and not in the original manuscript.

These various reorganisations and editorial changes result in an incomplete and somewhat idealised version of the original manuscript but they also produce a more readable story which brings new historical sources into the public domain for the first time. 